\title{
Tratamento tardio de fratura do complexo zigomático-orbitário com uso de fixação interna rígida
}

\author{
Late treatment of the zygomatic-orbital complex fracture with rigid internal fixation \\ Danyella Carolyna Soares dos Reis ${ }^{1}$ (D), Danilo Maldonado e Duarte ${ }^{1}$ (D), \\ Cristóvão Marcondes de Castro Rodrigues ${ }^{2}$ (D), Cláudia Jordão Silva ${ }^{3}$ (D), Flaviana Soares Rocha ${ }^{3}$ (D)
}

\begin{abstract}
RESUMO
As fraturas do complexo zigomático-orbitário são bastante frequentes devido a sua localização e projeção na face, podendo gerar grandes transtornos funcionais e estéticos ao paciente. O osso zigomático é essencial na configuração da face, sendo a principal estrutura formadora do terço médio dela. Os traumas que mais frequentemente provocam fraturas do complexo zigomático-orbitário são agressões físicas, acidentes de trânsito e esportivos. O tipo de fratura, tempo decorrido, a severidade e o envolvimento de outras estruturas faciais influenciam a modalidade de tratamento a ser empregado. O presente trabalho apresenta um caso clínico de fratura do complexo zigomático-orbitário esquerdo, diagnosticada tardiamente, e tratada por meio de osteotomia, redução e fixação em três pontos com placas e parafusos do sistema 1.5, e reconstrução do assoalho orbitário com tela de titânio.
\end{abstract}

Palavras-chave: Traumatismos Faciais; Ossos Faciais; Osso Zigomático; Órbita.

\begin{abstract}
Fractures of the zygomatic-orbital complex are quite frequent due to their location and projection on the face, which can cause major functional and aesthetic disorders to the patient. The zygomatic bone is essential in the configuration of the face, being the main forming structure of the middle third of it. The traumas that most often cause fractures of the zygomatic-orbital complex are physical aggression, traffic accidents, and sports. The type of fracture, elapsed time, severity, and the involvement of other facial structures influence the type of treatment to be employed. The present work presents a clinical case of fracture of the left zygomatic-orbital complex, diagnosed late, and treated by osteotomy, reduction, and fixation in three points with 1.5 system plates and screws, and reconstruction of the orbital floor with titanium mesh.
\end{abstract}

Keywords: Facial Trauma; Facial Bones; Zygomatic Bone; Orbit.

1. Faculdade de Odontologia, Universidade Federal de Uberlândia (UFU), Uberlândia (MG), Brasil.

2. Residência em Cirurgia e Traumatologia Buco-maxilo-facial, Faculdade de Medicina, UFU, Uberlândia (MG), Brasil.

3. Área de Cirurgia e Traumatologia Buco-maxilo-facial, Faculdade de Odontologia, UFU, Uberlândia (MG), Brasil.

$\bowtie$ Flaviana Soares Rocha. Universidade Federal de Uberlândia, Faculdade de Odontologia, Bloco 4T, Av. Pará, 1748 - Umuarama, Uberlândia (MG), Brasil. flavianasoares.rocha@gmail.com | Recebido em: 12/03/2019 | Aprovado em: 06/08/2019 


\section{INTRODUÇÃO}

O osso zigomático, localizado no terço médio facial, é a estrutura mais proeminente da face ${ }^{1-3} \mathrm{e}$, junto com o arco zigomático forma o complexo zigomático, importante estrutura que compõe o contorno facial. Devido a sua topografia e projeção na face, é altamente susceptível a traumas e lesões ${ }^{3,4,5}$. Os traumas que mais frequentemente provocam fraturas do complexo zigomático-orbitário são agressões físicas, acidentes de trânsito e esportivos ${ }^{6,7}$. As fraturas do complexo zigomático podem gerar comprometimentos funcionais e/ou estéticos importantes ${ }^{1,8,9}$ em função do seu íntimo relacionamento com outras estruturas da face, como a órbita ${ }^{1,2,10,11}$, o que torna o tratamento essencia| ${ }^{8,12,13}$. Os principais sinais $\mathrm{e}$ sintomas identificados em uma fratura do complexo zigomático-orbitário são: parestesia do nervo infraorbitário, epistaxe, assimetria facial por afundamento da região zigomática, hiposfagma, edema e hematoma periorbital, degrau ósseo em região infraorbitária, pilar zigomaticomaxilar e a diplopia ${ }^{12,13}$. Os exames imaginológicos são essenciais para o diagnóstico e tratamento dessas fraturas. O exame radiográfico consiste na incidência de Waters e de Hirtz, sendo que na primeira observa-se a provável fratura do osso zigomático e velamento do seio maxilar; na segunda incidência pode-se notar a conformação anatômica do arco zigomático bilateralmente ${ }^{13,14}$. A tomografia computadorizada é considerada o padrão ouro para o diagnóstico de fraturas faciais, principalmente no terço médio da face, pois ela permite uma resolução de imagem superior, grande sensibilidade e especificidade, além de possibilitar a reconstrução tridimensional (3D) das imagens obtidas, auxiliando no planejamento cirúrgico ${ }^{15}$. O tempo decorrido após o trauma é de grande importância na indicação da melhor forma de tratamento, pois pode alterar a conduta a ser adotada ${ }^{16,17}$. O diagnóstico e tratamento precoces das fraturas de complexo zigomático-orbitário oferecem a melhor oportunidade para adequada restauração funcional e estética ${ }^{18}$. Entretanto, lesões não diagnosticadas ou trauma sistêmico concomitante, com risco de morte, podem atrasar o tratamento ${ }^{19,20}$.

A opção de abordar uma fratura do complexo zigomático-orbitário com redução aberta ou fechada ainda é controversa. A redução cirúrgica aberta seguida por fixação interna rígida proporciona maior segurança e estabilidade, diminuindo o índice de com- plicações pós-operatórias e possibilitando o rápido retorno do paciente as suas funções ${ }^{14,16,17}$. O grau de deslocamento da fratura e a presença de cominuição são fatores importantes para a indicação do tratamento aberto ou fechado ${ }^{8}$. Entretanto, mesmo fraturas pouco deslocadas podem resultar em déficits funcionais e/ou estéticos significativos, justificando a necessidade de uma exposição cirúrgica adequada da fratura ${ }^{3}$.

Este trabalho objetiva apresentar um caso de fratura do complexo zigomático-orbitário, diagnosticado tardiamente e tratada por meio de redução aberta, osteotomia e osteossíntese com placa e parafusos de titânio, juntamente com a reconstrução do assoalho orbital com tela de titânio.

\section{RELATO DO CASO}

Homem branco, de 26 anos de idade, compareceu ao Serviço de Cirurgia e Traumatologia Bucomaxilofacial do Hospital de Clínicas da Universidade Federal de Uberlândia, MG, relatando queixa estética após acidente automobilístico ocorrido há 80 dias.

Durante anamnese o paciente não referiu alterações sistêmicas. Ao exame clínico, observou-se assimetria facial, distopia e perda de projeção zigomática do lado esquerdo da face. À palpação, foi constatado degrau na região do rebordo infra-orbitário, arco zigomático e sutura fronto-zigomática esquerdos. O paciente não apresentava edema, hematoma, oftalmoplegia, diplopia ou enoftalmia, porém, relatava limitação na abertura bucal após o acidente (Figura 1A-D).

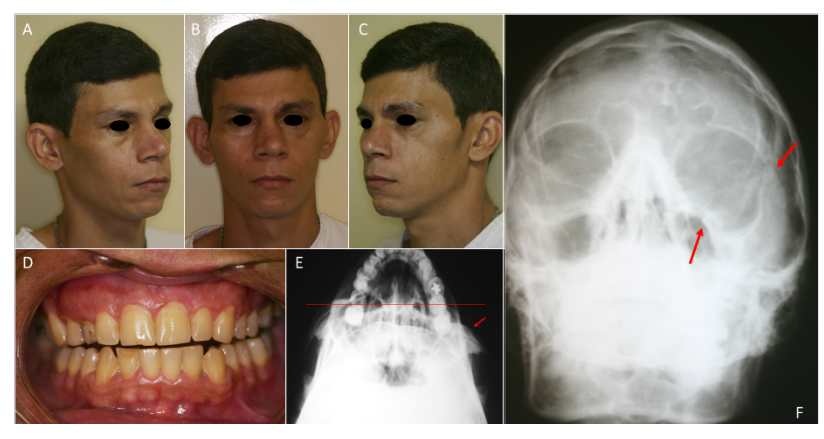

Figura 1: Aspecto clínico inicial: Perfil $45^{\circ}$ direito (A); Anterior (B); Perfil $45^{\circ}$ esquerdo (C); limitação de abertura bucal (D); Exames radiográficos em incidências de Hirtz (E) e Waters (F) confirmando o diagnóstico de fratura do complexo zigomático esquerdo. 
Foram solicitados exames radiográficos em incidências de Hirtz (submento vértice) e Waters (mento-naso), que evidenciaram a fratura do arco zigomático e a perda importante de projeção do complexo zigomático, além de descontinuidade óssea na região da sutura frontozigomática e margem infraorbitária esquerda, e alteração do contorno da órbita esquerda (Figura 1E-F). As imagens radiográficas foram complementadas com tomografia computadorizada de face e reconstrução tridimensional (Figura 2), evidenciando a fratura do complexo zigomático-orbitário e arco zigomático do lado esquerdo, impedindo o adequado movimento da mandíbula.

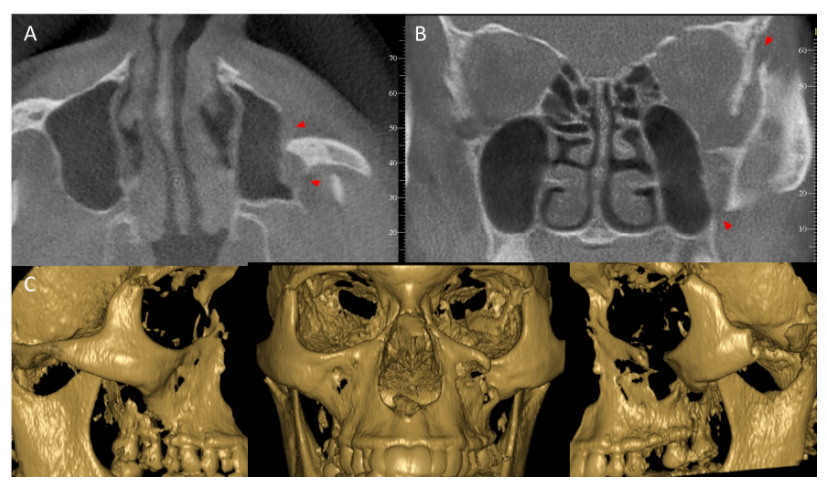

Figura 2: Imagens tomográficas em cortes axial (A) e coronal (B); Reconstrução tridimensional a partir das tomografias computadorizadas: Visão lateral direita (A) frontal (B) e lateral esquerda (C).

O paciente foi submetido ao procedimento cirúrgico para redução e fixação das fraturas sob anestesia geral, após 90 dias do acidente. Devido ao excesso de tempo decorrido entre o acidente e o diagnóstico das fraturas, bem como a consolidação da fratura em posição desfavorável, foi planejada a osteotomia de todos os pontos de fixação do complexo zigomático com auxílio de cinzel e martelo, excetuando-se a região de pilar zigomático-maxilar, onde havia perda de parte da parede anterior do seio maxilar. Para isso, foi realizado acesso cirúrgico em três pontos, sendo eles: acesso ao rebordo infraorbitário, à região da sutura fronto-zigomática e do arco zigomático. Foi realizada a incisão da pele, seguida pela divulsão dos tecidos até exposição completa do tecido ósseo. A redução e fixação das fraturas foi realizada utilizando-se uma osteossíntese com fio de aço na região infraorbitária e placas de titânio do sistema $1.5 \mathrm{~mm}$ na região da sutura fronto-zigomática e arco zigomático. Após exploração cirúrgica do assoalho orbitário, foi evidenciado perda de segmentos ósseos, o que poderia resultar em perda de suporte ao conteúdo ocular, sendo colocada uma tela de titânio para reposicionamento destas estruturas. A sutura foi realizada em planos com monocryl 4-0 e sutura em pele com Nylon 5.0 (Figura 3).

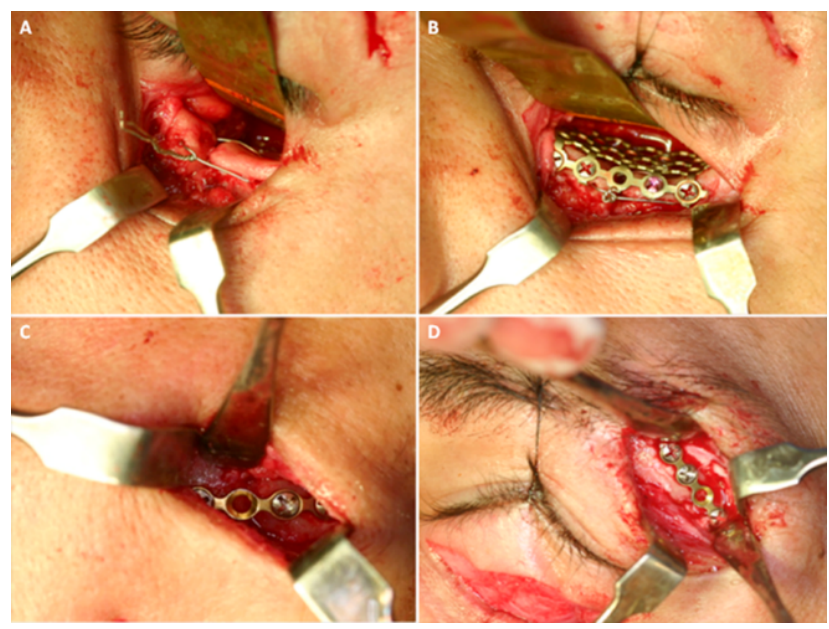

Figura 3: (A) Redução e osteossíntese com fio de aço; (B) Reconstrução do rebordo e assoalho orbital com tela de titânio; (C) Redução e fixação do arco zigomático com placa e parafusos; (D) Redução e fixação da sutura fronto-zigomática através de placa e parafusos.

O paciente permaneceu em acompanhamento ambulatorial, apresentando boa cicatrização e nenhuma intercorrência pós-operatória. No retorno ambulatorial com 53 dias de pós-operatório, apresentou manutenção da função ocular, melhora significativa na simetria facial e projeção adequada do complexo zigomático-orbitário esquerdo (Figura 4), recebendo alta ambulatorial. 


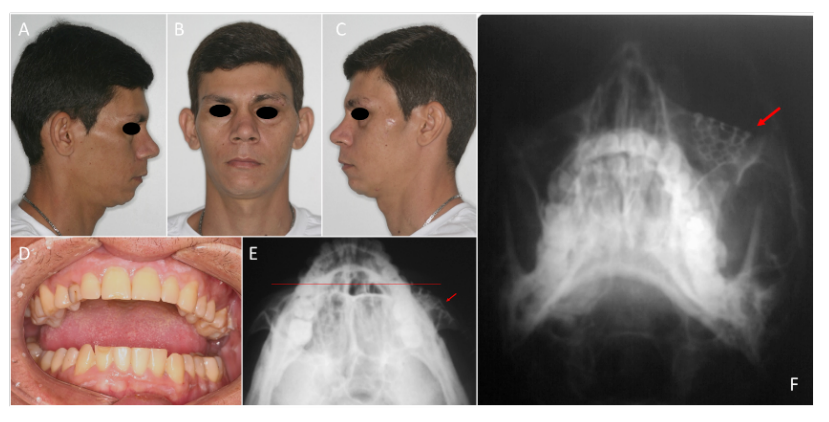

Figura 4: Aspecto clínico pós-operatório: Perfil direito (A); Anterior (B); Perfil esquerdo (C); Abertura bucal satisfatória (D); Exames radiográficos em incidências de Hirtz (E) e Waters (F) mostrando simetria dos rebordos infraorbitários e reposicionamento do osso zigomático.

\section{DISCUSSÃO}

O complexo zigomático-orbitário é uma estrutura importante do contorno facial. Devido a sua localização, está mais sujeito ao trauma do que outros ossos da face $^{3}$, exceto os ossos nasais $^{1,4,20}$. O complexo zigomático quando lesionado, pode trazer prejuízos funcionais e estéticos importantes, como perda de traço facial, limitação de abertura bucal, e alterações oculares e sensoriais ${ }^{1,9,21}$. Devido à existência do pilar zigomaticomaxilar, área de reforço ósseo, a energia do trauma direcionada ao zigomático é dissipada para as áreas mais frágeis que se articulam a ele, que tendem a fraturar, como o assoalho de órbita e a sutura fronto-zigomática ${ }^{7,20}$.

Por se relacionar intimamente com várias estruturas da face, quando ocorre lesão do complexo zigomático é essencial uma redução e fixação estáveis para uma reabilitação adequada ${ }^{12,13}$. Devido a sua morfologia complexa e posicionamento tridimensional, existe a dificuldade de reposicionamento do osso zigomático, o que pode acarretar achatamento malar, aumento da largura facial e degrau ósseo palpável, comprometendo a estética facial ${ }^{1,22}$.

Muitas técnicas de tratamento com diferentes índices de sucesso têm sido propostas na literatura para abordagem desse tipo de lesão $0^{14,16,18}$. Os sistemas de fixação com uso de miniplacas e parafusos tornou os métodos anteriormente utilizados, como fixadores externos, fios de aço e o tamponamento sinusal, formas obsoletas de tratamento, devido à instabilidade tridimensional ao osso zigomático. O fato das forças musculares que agem sobre o terço médio da face serem irrelevantes possibilita o uso de placas mais delgadas. As telas de titânio pré-fabricadas têm sido utilizadas para reconstrução do assoalho de órbita, com resultados satisfatórios na restauração do volume orbital 22 .

Alguns autores acreditam que a redução aberta pode desencadear um maior número de complicações em comparação com as técnicas de redução fechada ${ }^{23}$. Ainda assim, a fixação interna rígida é cada vez mais comum ${ }^{10,12,24}$, sendo considerada como tratamento ideal para estas lesões por possibilitar estabilidade adequada para o reparo ósseo ${ }^{2,17}$. As complicações das fraturas zigomáticas podem ser decorrentes das incisões de partes moles peri-orbitárias, e também do deslocamento dos cotos ósseos, como alterações do nervo infraorbitário, diplopia, enoftalmia, não união das fraturas, infecções ${ }^{4,12}$, limitação de abertura bucal e assimetria facial ${ }^{2,7}$. O refinamento da técnica cirúrgica, correto diagnóstico e plano de tratamento, são fatores que podem minimizar tais complicações, como no caso apresentado.

O tratamento das fraturas zigomático-orbitárias parece exibir melhores resultados quando realizado precocemente $7,18,20$. Entretanto, o edema e o hematoma periorbitários, podem dificultar o exame apurado dessas fraturas ${ }^{19,25}$. Além disso, politraumas podem necessitar de atendimento emergencial, postergando as fraturas do complexo zigomático-orbitário para a avaliação secundária do trauma.

A dificuldade de redução de tais fraturas é tempo-dependente devido à maior possibilidade de formação de tecido cicatricial osteoide ou má união dos cotos ósseos, sendo necessário osteotomia para reposição e fixação adequada da fratu$\mathrm{ra}^{25}$. Carr \& Mathog ${ }^{18}$, em 1997, verificaram que, em fraturas envolvendo o complexo zigomático e a orbita é possível uma boa redução primária até 21 dias após o trauma. Mesmo nessas situações a fixação interna rígida pode ser realizada com restauração completa da simetria facial, sendo considerada método ideal de tratamento de fraturas tardias ${ }^{20}$.

A combinação de múltiplos acessos possibilita a visualização direta dos pilares de sustentação do complexo zigomático ${ }^{2}$ assistindo no 
reposicionamento e fixação em local adequado. A associação do acesso cirúrgico do rebordo infraorbitário e sutura fronto-zigomática foi utilizada no presente caso para permitir melhor visualização, redução e a fixação em dois pilares do complexo zigomático ${ }^{10}$.

\section{CONCLUSÃO}

As fraturas que envolvem o complexo zigomático-orbitário estão entre as mais comuns da face. Os exames clínico e de imagem são indispensáveis para um correto diagnóstico e escolha de tratamento das fraturas do complexo zigomático-orbitário. Os acessos extra e intra-oral são suficientes para redução e fixação adequada das fraturas, mesmo que tardiamente diagnosticadas. A fixação interna rígida proporciona excelentes resultados do ponto de vista estético e funcional, continuando a ser o melhor método de escolha para tratamento.

\section{REFÊRENCIAS}

1. Kim J, Kim S, Chung S, Chung KY. Zygomatic Arch Fracture: A New Classification and Treatment Algorithm With Epidemiologic Analysis. J Craniofac Surg. 2014;25(4):1389-92.

2. Peretti N, MacLeod S. Zygomaticomaxillary complexfractures: diagnosis and treatment. Curr Opin Otolaryngol Head Neck Surg. 2017;25(4):314-9.

3. Bogusiak K, Arkuszewski P. Characteristics and Epidemiology of Zygomaticomaxillary Complex Fractures. J Craniofac Surg. 2010;21(4):1018-23.

4. Dingman RO, Natvig P. Cirurgia das fraturas faciais. São Paulo: Ed.Santos, 3a Reimpressão, 2004.

5. Erol B, Tanrikulu R, Gorgun B. Maxillofacial fractures. Analysis of demographic distribuition and treatment in 2901 patients (25- year experience). J Craniomaxillofac Surg. 2004;32:308-13.

6. Paulesini Junior W, Farias LP, Aquati M, Rapoporat A, Leporace AA. Fratura de Complexo Zigomático : Relato de caso. Revista de Odontologia da Universidade Cidade de São Paulo 2008;20(3):301-6

7. Hwang K, Kim DH. Analysis of Zygomatic Fractures. J Craniofac Surg. 2011;22(4):1416-21.

8. Buck DW 2nd, Heyer K, Lewis VL Jr. Reconstruction of the zygomatic arch using a mandibular adaption plate. J Craniofac Surg. 2009;20(4):1193-6.

9. Ellis III E, Zide MF. Surgical approaches to the facial skeleton. Baltimore: Ed. Willians \& Wilkins, 1997.
10. Becelli R, Quarato D, Matarazzo G, Renzi G, Dominici C. Esthetic positioning of rigid internal fixation in tripod zygomatic fractures: an innovative surgical technique. J Craniofac Surg. 2009;20(3):724-5.

11. Teixeira LMS, Reher P, Reher VGS. Anatomia aplicada à odontologia. Rio de Janeiro: Guanabara Koogan, 2001. 372 p.

12. Fonseca RJ, Walker RV. Oral and maxillofacial trauma. Philadelphia: Ed. W. B. Saunders Company, 2a ed., v. 2, 1997.

13. Makowski GJ, Van Sickels JE. Evaluation of results with three-point visualization of zygomaticomaxillary complex fractures. Oral Surg Oral Med Oral Pathol Oral Radiol Endod. 1995;80(6):624-8.

14. Kloch DW, Gilliland R. Internal fixation versus conventional therapy in midface fractures. J Trauma. $1987 ; 27(10): 1136-45$.

15. Strong EB, Gary C. Management of zygomaticomaxillary complex fractures. Facial Plast Surg Clin N Am. 2017;25:547-62.

16. Sands T, Symington O, Katsikeris N, Brown A. Fractures of the zygomatic complex : a case report and review. J Can Dent Assoc. 1993;59(9):749-55.

17. El-Anwar MW, Elsheikh E, Hussein AM, Tantawy AA, Abdelbaki YM. Transconjunctival versus subciliary approach to the infraorbital margin for open reduction of $z y-$ gomaticomaxillary complex fractures: a randomized feasibility study. Oral Maxillofac Surg. 2017;21:187-92.

18. Carr RM, Mathog RH. Early and delayed repair of orbitozygomatic complex fractures. J Oral Maxillofac Surg. 1997;55:253-8.

19. Whitaker LA, Yaremchuk MJ. Secondary reconstruction of posttraumatic orbital deformities. Ann Plast Surg. $1990 ; 25(6): 440-9$.

20. Lu W, Zhou H, Xiao C, Shen Q, Lin M, Fan X. Late Correction of Orbital-Zygomatic-Maxillary Fractures Combined With Orbital Wall Fractures. J Craniofac Surg. 2012;23(6):1672-6.

21. Kaukola L, Snäll J, Roine R, Sintonen H, Thorén H. Health-related quality of life of patients with zygomatic fracture. Med Oral Patol Oral Cir Bucal. 2017;22(5):636-42.

22. Ellstrom CL, Evans GRD. Evidence-Based Medicine: Zygoma Fractures. Plast Reconstr Surg. 2013;132(6):1649-57.

23. Moreno EFC, Vasconcelos BCE, Carneiro SCAS, Catunda IS, Melo AR. Evaluation of Fixation Techniques With Titanium Plates and Kirschner Wires for Zygoma Fractures: Preliminary Study. J Oral Maxillofac Surg. 2012;70:2386-93.

24. Dziadek $\mathrm{H}$, Cieślik T. Treatment of zygomatico-orbital and zygomatico-maxillo-orbital fractures by open reduction and rigid internal fixation. Wiad Lek. 2005;58 $(5-6): 270-4$.

25. Paulesini Junior W, Farias LP, Aquati M, Rapoporat A, Leporace AA. Fratura de Complexo Zigomático : Relato de caso. Revista de Odontologia da Universidade Cidade de São Paulo. 2008;20(3):301-6. 